\title{
METODOLOGÍA DEL TRABAJO SOCIAL CON INMIGRANTES Y REFUGIADOS
}

\author{
$M^{\mathrm{a}}$ DEL SOCORRO ESCOBAR RUBIO \\ NIEVES GASCÓN NAVARRO \\ Trabajadoras Sociales
}

\section{INTRODUCCIÓN}

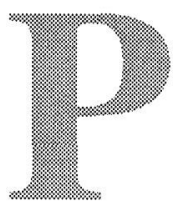

artimos de la existencia de un debate generalizado en torno a la metodología del Trabajo Social ${ }^{1}$. Esta discrepancia afecta también al trabajo con inmigrantes y refugiados. No vamos a profundizar sobre este tema para concluir en la falta de uniformidad metodológica, sino que, al margen, realizaremos una propuesta concreta avalada por nuestra experiencia metodológica y cotidiana en materia de intervención social con inmigrantes y refugiados. En este sentido, diferenciamos entre una metodología más tradicional del Trabajo Social, y otra que consideramos también factible, pero actualmente no es habitual su práctica en la Comunidad de Madrid.

- Respecto a la metodología tradicional distinguimos:

- Trabajo social individual o de casos: el trabajo parte de la demanda individual y a veces familiar. Dicha demanda es sentida y expresada por el interesado, o detectada por el trabajador social.

- Trabajo social de grupos y comunidad: parte de los conflictos y las necesidades de un grupo o una comunidad. No tiene que existir una demanda expresada por los interesados, aunque el conflicto grupal puede generar demandas individuales múltiples.

Consultar:

1. De la Red, Natividad. Aproximación al Trabajo Social. Edit. Consejo Gene, de Colegios Oficiales de Diplomado en TS y AASS. Madrid, 1993.

2. Escartín, Ma Joséy Suarez Soto, Esperanza. Introducción al Trabajo Social. Edit. Aguaclara. Alicante, 1994

3. Juarez, Miguel; Gaitán Lourdes; Urosa, Belén y Cabrera, Pedro J. Trabajo Social e Investigación. Edit. Universidad Pontificia de Comillas. Madrid, 1993.

4. Payne, Malcon. Teorías contemporáneas del Trabajo Social. Ediciones Paidos S.A. Barcelona, 1992.

5. Zamanillo, Teresa y Gaitán, Lourdes. Para comprender el Trabajo Social. Edit. Verbo Divino. Madrid, 1991. 
- En torno a la forma de trabajo menos habitual en la práctica profesional, pero que consideramos igualmente factible, proponemos a continuación:

- INVESTIGACIÓN-ACCIÓN: a través de la investigación, hacemos participar a un grupo o comunidad en la organización conjunta de proyectos de trabajo.

Una metodología de caso puede acercarnos a las necesidades del grupo, por otro lado la metodología grupal nos puede conducir al Trabajo Social de casos. En cualquiera de los métodos a emplear, debemos tener en cuenta cuestiones previas como:

- Tipo de trabajo que debemos realizar, es decir, qué tipo de programa o proyecto se va a ejecutar.

- Función y sector desde el que trabajamos (sanidad, servicios sociales, educación, etc.).

- Las características específicas de los usuarios, en especial, debemos conocer las costumbres, cultura y usos propios del grupo o los grupos de inmigrantes y refugiados con los que trabajaremos, etc.

Cremos importante referir el tipo de ventajas y dificultades que son inherentes, a nuestro criterio, a los propios inmigrantes, y que son puntos claves a considerar por los profesionales que trabajamos con dichos grupos:

- Ventajas:

- son personas jóvenes, y generalmente se encuentran en edad laboral;

- encontramos una actitud favorable al cambio, el hecho de dejar el país de origen y emigrar a otro país con cultura y, en algunos casos, con idioma diferente, supone la ampliación de las expectativas de futuro y la posibilidad de propiciar un cambio de la forma de vida en general.

- Dificultades:

- este colectivo presenta una gran movilidad y dispersión geográfica;

- nos encontramos en numerosas ocasiones con situciones de irregularidad jurídica;

- existencia de diferencias culturales entre los distintos colectivos de inmigrantes, y entre éstos y la población autóctona;

- muchos inmigrantes y refugiados mantienen persistente la idea de «estar de paso» en la sociedad de acogida;

- en muchos casos, se observa y se mantiene una actitud de «hermetismo cultural», ya que, por una razón de identidad, viven más intensamente las costumbres y hábitos culturales de la sociedad de origen. 


\section{TRABAJO SOCIAL DE CASOS O INDIVIDUALIZADO}

Comprende las siguientes fases:

\subsection{Toma de contacto}

En esta etapa, la labor fundamental es la recogida de datos y, para ello, utilizamos las técnicas de la entrevista, semidirigida, básicamente, y la observación, a través de encuentros en el despacho del profesional o en el domicilio de los inmigrantes y refugiados. El objetivo fundamental es llegar al conocimiento de la situación social de la persona con la que trabajaremos.

Debido a la relevancia de algunos de los datos específicos de estos colectivos (el estatuto jurídico, conocimiento del castellano, grado de integración social, reagrupación familiar, etc.), y tras consultar distintos modelos de fichas empleadas, estudiando la tipología y clasificación de los datos más relevantes, hemos creído oportuno proponer un modelo concreto de ficha social que posibilite una mayor operatividad en la recogida de datos y posterior intervención profesional (ver anexo II).

Por último, y para completar esta etapa, es interesante establecer contacto con otros profesionales de otros organismos y sectores (educación, sanidad, etc.) que igualmente trabajen con estos colectivos, y con los que podamos intercambiar y recopilar más información a través, fundamentalmente, de entrevistas y reuniones, así como darnos a conocer y poder establecer posteriormente una coordinación interprofesional.

\subsection{Diagnóstico o valoración}

En esta fase, trataremos de organizar e interpretar los datos recogidos en la primera etapa.

Para realizar un diagnóstico adecuado, debemos tener en cuenta los aspectos peculiares del caso y documentarnos sobre las características culturales y formas de vida del colectivo al que pertenece el usuario, acudiendo a estudios realizados, o a bibliografía especializada, que podemos encontrar en centros de documentación (como los dependientes de la Dirección General de Migraciones, Ministerio de Asuntos Sociales, etc.).

Finalmente, señalamos importante considerar el marco institucional desde el cual estamos trabajando el caso, por las limitaciones que conlleva y por los recursos de los que disponemos. 


\subsection{Programación}

Debemos trabajar conjuntamente con el usuario. Expondremos nuestra valoración y trabajaremos la toma de conciencia, el grado de implicación del interesado y la motivación.

Es importante llegar a consensuar un programa de intervención con el usuario. En este sentido, vemos adecuado que se produzca una buena comunicación profesional-cliente; consecuentemente, es importante tener en cuenta el conocimiento del castellano de los inmigrantes, y por otro lado, la formación de los trabajadores sociales que los atienden. Es decir, nos referimos al conocimiento de idiomas, la experiencia profesional acumulada, y la formación en materia de inmigración y refugio, como puntos claves a la hora de conseguir resultados operativos profesionalmente. A todo ello, añadiremos la actitud, por parte del profesional, de tolerancia y de no prejuzgar a los usuarios.

\section{TRABAJO SOCIAL DE GRUPOS Y COMUNIDAD}

La aplicación de esta metodología al intervenir con la población inmigrante y refugiada, al igual que con la población española, no es excluyente. Las metodologías en Trabajo Social se pueden y se deben utilizar de forma complementaria, en función de la realidad que tenemos y de los objetivos que nos propongamos a corto y largo plazo.

En función del lugar desde el que intervengamos, nuestros cometidos pueden ser (entre otros) el desarrollo de un proyecto comunitario o, por el contrario, la atención de demandas individuales (trabajo de casos).

\subsection{La intervención comunitaria nos puede surgir}

1. A través de las demandas individuales: por la información que nosotros mismos recogemos en las atenciones individuales y que nos dan un análisis básico de necesidades/demandas, a las que nosotros nos propondríamos responder con una metodología de intervención comunitaria (proceso endógeno: surge de la labor cotidiana del propio profesional).

2. A través de un proyecto predeterminado por otros equipos, y entregados al trabajador social para que los lleve a cabo (exógeno: proceso impuesto por agentes externos). Un ejemplo concreto de este tipo de intervención podría ser el siguiente:

«La Comunidad de Madrid realiza un estudio sobre las condiciones socio-sanitarias de la población en general. El análisis de los datos 
recibidos ofrece una información genérica, tanto de la población autóctona como de la extranjera. La Comunidad se plantea intervenir para paliar esas necesidades detectadas, y establece las estrategias oportunas. De cara a la población extranjera, considera necesario trabajar en colaboración con $\mathrm{ONG}^{\prime}$, a las cuales llega un programa, con una información especifica en cuanto a extranjeros, general en cuanto a sus situaciones y con unos objetivos concretos.

La ONG pone en marcha el programa a través de un equipo en el que se cuenta con un trabajador social, pero, a la hora de llevarlo a la práctica, es necesario actualizar los datos y ampliarlos (análisis de la realidad), porque se partía de una detección general y debemos tener en cuenta que las variaciones en la población extranjera se producen con una frecuencia mucho mayor que en la población autóctona.

Siguiendo con nuestro ejemplo de metodologia de intervención comunitaria con grupos o comunidad desde un proceso endogeno, en cuanto a los destinatarios, normalmente se trata al «colectivo» sin especificar y, en nuestro caso, las diferencias en cuanto a situación legal, orígenes, nacionalidad, religión, idioma, dispersión y movilidad geográfica son condicionantes claros al tiempo de iniciar e implementar una intervención comunitaria.

Un ejemplo ilustrativo: Si en un programa de vacunaciones con la población extranjera, deseamos cumplir nuestros objetivos y calendario, debemos tener en cuenta que, por ejemplo, la población polaca difiere por completo de la población marroqui (el sistema sanitario de procedencia es diferente, también los hábitos, etc.).

Siguiendo el mismo ejemplo, en cuanto a la población marroquí, nuestros objetivos no se cumplirán:

1. Si desconocemos las características y fechas del Ramadan. EI Ramadán dura treinta días, durante ese tiempo no se puede ingerir ni introducir sustancias externas en el organismo hasta la puesta de sol.

2. Si ponemos las atenciones a las 12 de la mañana de lunes a viernes. Se programan las atenciones en horario de mañanas en días laborables exclusivamente. Los inmigrantes y refugiados que trabajan lo hacen en ese periodo de tiempo, por lo que no pueden acudir. Además, muchos no pueden pedir permisos por estar empleados de forma irregular o tener miedo a que el empleador se entere de su posible estado de salud y pierda su puesto de trabajo.

3. Si esperamos encontrar al $100 \%$ de la población al año siguiente para realizar un seguimiento y evaluación. Problema de movilidad geográfica.

Una vez finalizado nuestro ejemplo, es obvio que, en la práctica, se observa que al venir dados los proyectos «desde arriba» (exógenos), 
las necesidades que se van detectando por su implementación son mayores o diferentes, y se corre el riesgo de crear falsas expectativas si nos adelantamos con nuestra oferta a la demanda de los destinatarios.

Por todo lo dicho anteriormente y los ejemplos expuestos, es obvio que la evaluación, actualización de datos y retroalimentación deben ser constantes en el trabajo con la población extranjera. Por ello, proponemos un establecimiento de objetivos, temporalización y evaluación, acordes al objeto de nuestra intervención.

\subsection{Fases}

Esta metodología contiene unas fases comunes en todo tipo de intervención social a realizar con diferentes colectivos, que son las siguientes:

$1{ }^{a}$ Diseño del proyecto de intervención a partir de la detección de necesidades. Establecimiento de hipótesis.

2. ${ }^{a}$ Toma de contacto y análisis de la realidad: en esta etapa verificaremos las hipótesis previamente planteadas a través de entrevistas con profesionales y de contactos con líderes naturales de la comunidad inmigrante y de refugiados; también debemos darnos a conocer y presentar el proyecto de intervención que vamos a llevar a cabo.

3. ${ }^{a}$ Retroalimentación: recibiremos y daremos información que debemos contrastar y comparar con la que teníamos preliminarmente.

4. ${ }^{a}$ Definición de técnicas a utilizar, escogiendo las más adecuadas a la realidad y características socioculturales del grupo o grupos de inmigrantes y refugiados.

5. ${ }^{a}$ Diseño de actividades, adecuando y consensuando éstas con el tipo de intereses y necesidades detectadas y planteadas por los propios inmigrantes.

6. ${ }^{a}$ Temporalización: es importante recordar que, cuando trabajamos con algunos grupos inmigrantes y refugiados, sobre todo en el caso de colectivos negroafricanos y marroquíes, el concepto del tiempo, su abstracción y previsión mental son totalmente distintos a los de la cultura española. Las fechas de siembra, recolección, u otras importantes por motivos religiosos, marcan los periodos de temporalización. Esta característica es básica a la hora de preveer un período de tiempo determinado para la ejecución de un proyecto, para lo cual proponemos necesario dedicar más tiempo del que normalmente solemos utilizar con otro tipo de población.

7. ${ }^{a}$ Previsión de recursos a emplear: en este apartado nos parece importante señalar la relevancia de la conyuntura sociopolítica actual en relación al tema de la inmigración, y debido a la numerosa presencia de ONG's, de programas, proyectos y subvenciones que faci- 
litan todo un despliegue de medios y recursos existentes destinados a favorecer a dicho colectivo. A nuestro juicio, debemos aprove-char este momento y articular los recursos de una manera coordinada y profesional.

8. ${ }^{a}$ Evaluación y seguimiento: ambos deben ser constantes cuando trabajamos con población inmigrante, para ir adecuando en todo momento la intervención a la realidad constantemente cambiante de este colectivo.

\section{UNA PROPUESTA: LA INVESTIGACION-ACCION-PARTICI- PANTE}

«La investigación, (según señala Lourdes Gaitán) como proceso de busqueda de saberes y conocimiento, no ha sido ajena al Trabajo Social» (Juárez, M. y Gaitán, L.: Trabajo Social e Investigación.Madrid, 1993). El Trabajador Social observa las diferentes demandas y recursos con los que trabaja habitualmente, a la vez que analiza sus propias necesidades como profesional, por lo que, al intervenir, entra en juego la función del Trabajador Social (TS), olvidada desde la última déca$\mathrm{da}$, como generador de recursos encaminados a paliar unas necesidades concretas, en principio, y, por otro lado, a detectar nuevas demandas/necesidades, puesto que no toda la población extranjera es la que llega a este profesional.

El TS decide en ese punto iniciar/continuar lo que denominaríamos Investigación-Accion-Participante (IAP) desde el Trabajo social.

El Trabajador Social es el profesional más adecuado porque es quien, por norma, está informado de la situación global del extranjero en el aspecto social, sanitario, laboral, legal, escolar, etc. Es quien, a su vez, parte de una formación multidisciplinar y, por tanto, es capaz de organizar y trabajar en un equipo compuesto por profesionales de diferentes disciplinas. Además, es él quién está antes, durante y después de detectar las demandas.

«La IAP es una aproximación para reducir la amplia separación entre los que tienen y los que no tienen» (Camplens, 1990). Uno de los aspectos de la investigación-acción participativa es que trata de reducir la distinción entre el investigador y el investigado, incorporando a ambos en un esfuerzo cooperativo de creación cognitiva: «proceso encaminado a adquirir conocimientos» (Rapaport, 1985; Bookman y Margen, 1988. Gaventa en prensa) ${ }^{2}$.

Sarri , Rosemary y Sarri, Catherine: «La investigación-acción participativa en dos comunidades de Bolivia y Estados Unidos». Traducción Colegio Diplomados Trabajo Social y AASS. Madrid, 1996. 
¿Para qué nos puede servir la IAP con población inmigrante y refugiada?

Para identificar necesidades comunitarias y desarrollar proyectos para tratar esas necesidades de manera cooperativa.

¿Qué pretenden?

Asentar las bases para la participación, capacitando a los beneficiarios para identificar y tratar sus necesidades básicas.

¿Cómo se lleva a cabo?

- Incorporando miembros de la comunidad afectados por los problemas a la participación activa.

- Combinando estrategias de investigación e intervención

- Construyendo destrezas locales y de liderazgo para apoyar los cambios.

- Facilitando el desarrollo de las relaciones laborales cooperativas entre agencias de servicios, investigadores y residentes comentarios.

Desde nuestra experiencia con población refugiada y/o inmigrante, entendemos que las estrategias de la IAP son especialmente convenientes para el colectivo extranjero y, más en concreto, para el de inmigrantes. Colectivos que tienen limitada su participación, no ya sólo en política, sino en economía e, incluso, en actividades sociales. Presentan, además, en su mayoría, un gran desconocimiento de la realidad española y desconfianza en las administraciones locales, etc., así como en otros organismos (fuerzas de seguridad, centros médicos), y cierta incapacidad, aunque cada vez menos, para aunar ideas o criterios y, por lo tanto, demanda de ayudas o recursos.

Por otro lado, si contamos con que en el colectivo de la población extranjera, en un elevado porcentaje, puede hablarse de nivel educativo medio, y tiene una gran voluntad y, por contra, ofrece poco rechazo al cambio, puede ser, y es, un recurso humano importantísimo para, en colaboración con el TS, especificar las necesidades no descubiertas (individuales/colectivas) y construir la capacidad de la comunidad para reconocer las causas de esas necesidades comunitarias y desarrollar e implementar esfuerzos colectivos para tratarlos. Creando así una relación de colaboración entre el propio investigador, en este caso el TS, y los inmigrantes y/o refugiados que pudiera facilitar el co-aprendizaje y la colaboración.

La IAP, en el contexto del desarrollo comunitario con población extranjera, es una alternativa para la intervención del Trabajo Social. Activa el cambio social sostenido porque el nivel de intervención se da más sobre la comunidad que sobre el individuo. Con la población extranjera, hasta ahora, la mayoría de las intervenciones se orientan a trabajar desde la perspectiva del caso o de comunidad, pero en contadas ocasiones se orienta a los profesionales de las instituciones, ONG's, a iniciar a través de una básica detección de necesidades, a una adecuada IAP. 


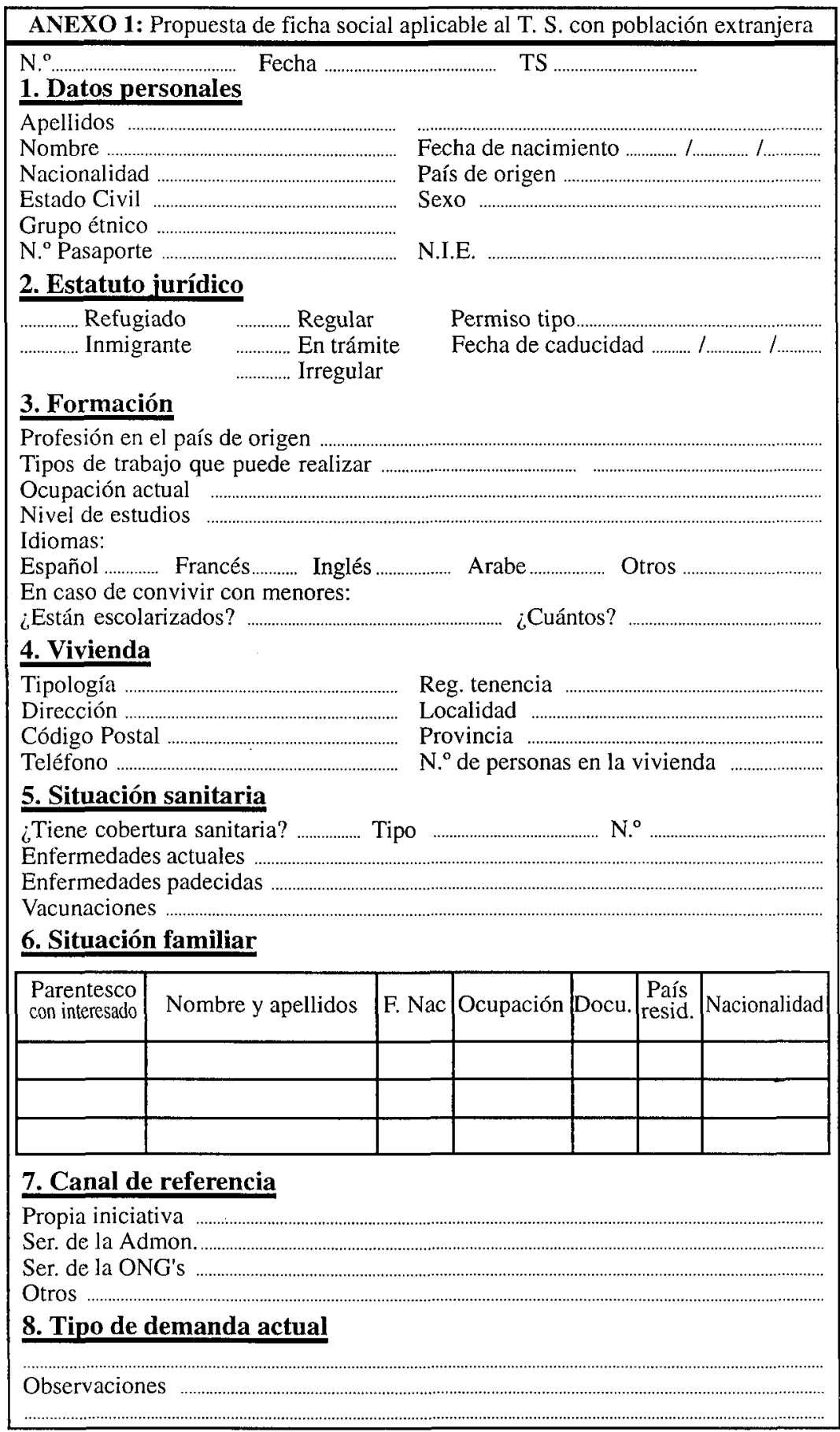




\subsection{Fases de la Investigación-Acción-Participante}

A continuación, exponemos las fases de la IAP desde un modelo teórico, y las variables a estudiar en la población inmigrante y refugiada.

Fases de la I.A.P.

- Informe de necesidades:

Metas:

- Especificar las necesidades.

- Construir la capacidad de la comunidad.

- Crear una relación de colaboración entre instituciones y miembros de la comunidad.

Técnicas:

- Observación participante.

- Examen ciudadano (concienciación y motivación).

- Foro comunitario (reuniones).

- Entrevistas semiestructuradas (con población autóctona y extranjera, profesionales, etc.).

- Planificación de la Acción:

En función de las necesidades halladas y las preliminares planteadas.

- Desarrollo de la acción:

Implementación del proyecto.

- Resultados del Proyecto:

Examen en cuanto a las metas, planificación y devenir. 
ANEXO II: VARIABLES PARA EL ESTUDIO Y CONOCIMIENTO DE NECESIDADES Y RECURSOS DE LA POBLACIÓN EXTRANJERA.

1. Inmigrantes

1.1. Variables:

- Origen y evolución del colectivo.

- Tamaño del colectivo y composición por sexos y edad.

- Vivienda y núcleo de convivencia.

- Actividad: trabajo y paro.

- Documentación.

- Situación sanitaria.

- Redes comunitarias.

- Mantenimiento de pautas culturales propias y relaciones con el lugar de origen.

2. Emigrantes:

2.2. Variables:

2.2.1. Demográficas:

- Distribución por sexo y edad.

- Estructura de edades.

- Población infantil.

- Estado civil.

2.2.2. Familia:

- Tipo de familia predominante y tamaño familiar.

- Composición.

- Matrimonios interétnicos.

2.2.3. Situación educativa y nivel de instrucción:

- Grado de escolarización de la población de 4 a 14 años.

- Tipo de centro al que acuden.

- Desfase escolar que presentan los alumnos.

- Nivel de instrucción de la población mayor de 14 años.

2.2.4. Situación laboral:

- Estructura de la actividad económica.

- Carácter y tipología del trabajo.

- Actividad laboral básica de la familia.

2.2.5. Situación sanitaria:

- Frecuentación médica.

- Frecuentación hospitalaria.

- Cobertura sanitaria.

- Lugar al que acuden cuando se ponen enfermos.

- Grado de satisfacción con la atención recibida.

- Enfermedad mental.

2.2.6. VIvienda. 